\title{
Tracking for inspection in energy transmission power lines using unmanned aerial vehicles: a systematic review of current and specific literature
}

\author{
Wander M. Martins ${ }^{1}$, Antonio J. Dantas Filho² ${ }^{2}$ Leandro D. de Jesus ${ }^{3}$, Adler D. de Souza ${ }^{4}$, \\ Alexandre C. B. Ramos ${ }^{5}$, Tales C. Pimenta ${ }^{6}$ \\ ${ }^{1,6}$ Institute of Engineering, Systems and Information Technology, Federal University of Itajuba, Brazil \\ ${ }^{2,3,4,5}$ Institute of Mathematics and Computation, Federal University of Itajuba, Brazil
}

\begin{tabular}{l}
\hline \hline Article Info \\
\hline Article history: \\
Received Mar 22, 2020 \\
Revised Apr 29, 2020 \\
Accepted Aug 18, 2020 \\
\hline
\end{tabular}

Keywords:

Autonomous

Simulation

Systematic review

Tracking

Transmission lines

UAV

\begin{abstract}
Power transmission lines are of great importance for the operation of all sectors of society, such as commerce, industry and public agencies. To ensure reliability and availability of power supply, regular and occasional inspections are conducted, mostly using patrol with binoculars, helicopters, or truck cranes. Research is being developed using unmanned aerial vehicle (UAV) to make this activity autonomous, faster, safer, and less costly. The present work aims to analyze research related to the autonomous control of the UAV along the transmission lines through a systematic review of the literature (SRL), apply a viable solution and to verify the possible lacuna in this state of the art. Improvements in safety, computational process and energy efficiency with low-cost were identified. The results presented can help the research community to perform the working this state of art, from the suggestions of autonomous tracking of transmission lines.
\end{abstract}

This is an open access article under the CC BY-SA license.

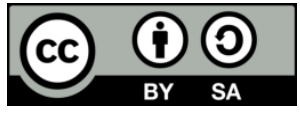

\section{Corresponding Author:}

Wander M. Martins,

Institute of Engineering, Systems and Information Technology,

Federal University of Itajuba, Itajuba, Brazil,

1303, Ave. B P S, Pinheirinho, Itajubá, Minas Gerais, Brazil, 37500-903.

Email: wandermendes@unifei.edu.br

\section{INTRODUCTION}

According to National Electrical System Operator (ONS), a Brazilian private company responsible for coordinating and controlling the operation of electricity generation and transmission facilities in the National Interconnected System (SIN) and for planning the operation of isolated systems in Brazil, in 2023 the extension of electricity transmission lines in this country will be $185,484 \mathrm{~km}$ [1]. The transmission structure is subject to damages of the nature actions and the high age, necessitating, periodically or after great storms, thorough verification. Inspection of high voltage power transmission lines is carried out in most cases by manned aerial vehicles or foot patrol.

The inspection by foot patrol is performed by electricians, located on platforms in the place with the aid of lifting trucks, or platforms isolated with the aid of binoculars [2]. In the inspection method by helicopter, a flight is carried out parallel to the transmission line and an operator performs the filming of the line. These methods, although functional, have many disadvantages, highlighting the risk of exposure of workers and the high financial cost per course. The development of unmanned aerial vehicle (UAV) or remotely piloted aircraft (RPA) technology has already been applied to many traditional civilian and military applications. One possible application is the replacement of traditional means of inspecting power transmission lines, however new issues are raised, among them the way to perform with lower cost, better performance, and safety. 
The aim of this work is to identify in state of the art, in publications the best way to perform power transmission line tracking using UAV. For this, the systematic literature review (SRL) process was used, verifying in the academy how this problem is being treated and providing a technical analysis for the solution. The SRL applies a well-designed research protocol, to search for articles in scientific databases, and with result of the analysis, answer the research questions. This study aims to assist other researchers and professionals in verifying the methods used, supporting decision making in future projects. There is great importance in the periodic review of these facilities, both for society, and for the possibility of automating the process offering low cost and high efficiency, thus providing the stability of the energy service.

To assist in the development of this study, the Parsifal [3] tool was used. It has the main steps necessary for the development of SRL, in addition to enabling online collaboration of the authors and the generation of analytical reports. Thus, an extensive material is produced, with all the necessary information of the articles, to synthesize the result in this work.

This paper is structured as follows. Section 2 describes the research methodology used in this work, the processes performed, and the research questions to be answered from this systematic review. Section 3 presents the analysis of the selected articles and their classifications. Section 4 presents the results obtained in the extraction. Section 5 there was a discussion of the results regarding the research questions and the last section presents the conclusion of this work.

\section{RESEARCH METHOD}

The methodology chosen for this study follows the detailed protocol originally proposed by Kitchenham [4] presented in Figure 1. This model has a methodologically well-defined research process to be applied that identifies, analyzes, and interprets available evidence related to research questions.

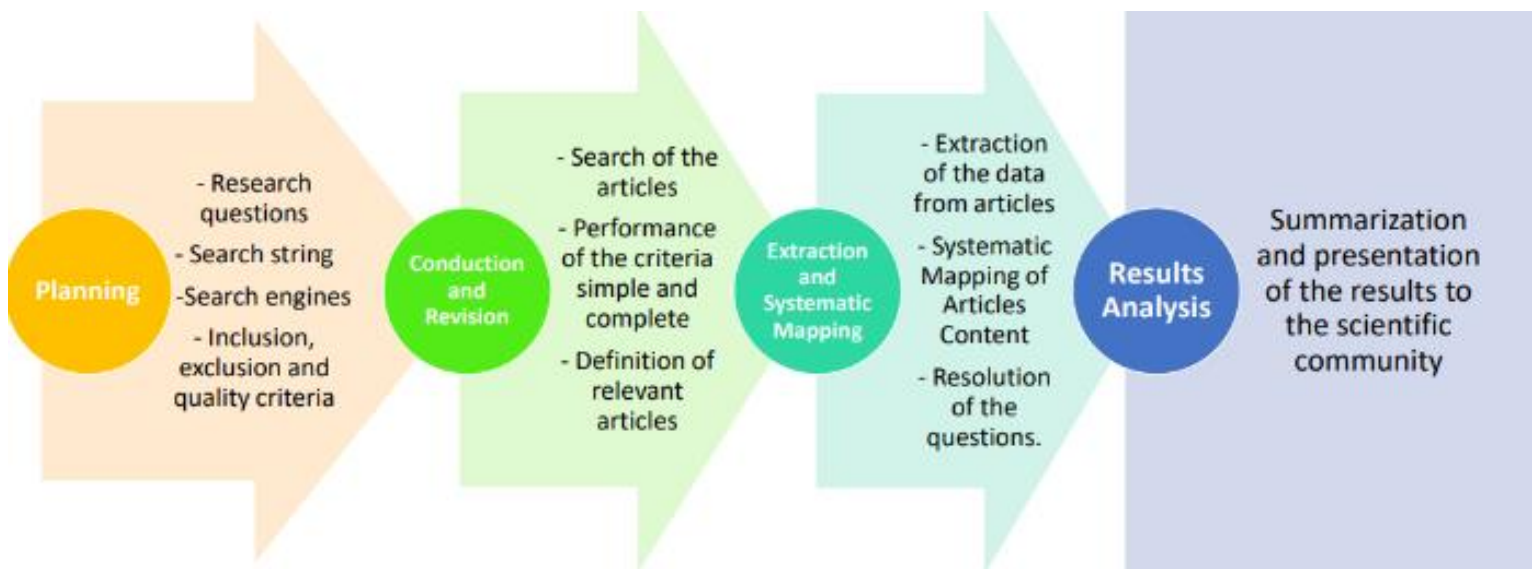

Figure 1. Steps for the development of a systematic review of literature

\subsection{Research definition}

The objective of this work is to analyze UAV guidance techniques for the inspection of power transmission lines and to identify materials and methods used in the autonomous transmission lines inspection from the point of view of the researchers in the area in the academic context. The elaboration of the question is of paramount importance for the outcome of the SRL. The answers to these questions make possible the specific analysis to determine the initial focus of the research and make possible the contribution to the academic environment. Thus, the following main question (MQ) is defined:

- What are the best techniques used to trace transmission lines in an automated, safe and low-cost way?

The MQ seeks to obtain the best way to perform the transmission line tracking and, for this, it takes into consideration important aspects such as efficiency, safety and cost.

From the MQ, specific questions (SQ) are defined in a secondary way:

- SQ1: What is the cost of implementing the materials and techniques used to inspect the transmission lines?

- SQ2: What is the computational and energetic efficiency of the techniques used to guide the UAV?

- SQ3: What is the level of security and the locations of the implemented solutions for the tracking of transmission lines?

Int J Rob \& Autom, Vol. 9, No. 4, December 2020 : 233 - 243 
Subsequently, the evidence will be materialized in published primary articles retrieved from selected research sources. The retrieval of these works happens through a search string (SS) that summarizes the questions to be searched. Thus, criteria were defined to guarantee the balance, feasibility and accessibility of the information for the execution of the study through this string, the criteria for conduction are:

a. Possessing search engine that allows the use of logical expressions or equivalent mechanism, returning the expected from the string.

b. Include in its collection with relevance in the academic environment in the areas of computing and engineering.

c. The search engines should allow search in the full text of the publications.

d. Own publication date between January 1, 2014 and June 1, 2019.

e. It should have the feature of filtering only article.

f. The publication must be available in the English language.

The research was conducted from four digital libraries; they are Association for Computing Machinery (ACM) (dl.acm.org), Institute of Electrical and Electronic Engineers (IEEE) (www.iee.org), Scopus (www.scopus.com) and Compendex (www.engineeringvillage.com). These bases were chosen by the experiment carried out by Dybala et al. [5]. As argued above, some databases currently known as Google Scholar, PubMed, and dissertation and theses libraries have not been considered, as they do not meet the driving criteria cited. However, it is known that most of the articles contained in these platforms are also available in the selected bases, as these are from the field of study and are of high academic relevance.

\subsection{Search terms}

In the context of the study objective, this work defines a strategy to construct the SS, this string is represented generally by the keywords related to the search questions and that can be used in the search. The strategy included:

a. Identification of keywords considering the main research question.

b. Identification of keywords considering the control article [6] originated from the research group.

c. Synonyms identified in the context.

The main search expression needs specific adjustments in the string to meet the driving criteria. Following the reapplication of the strategy of construction and refinement of the string the final expression used was: ("Autonomous" OR "Self-Navigation" OR "Self Navigation" OR Automatic OR AutoPilot) AND ("Remotely Piloted Aircraft" OR RPAS OR ARP OR "Unmanned Aerial Vehicle" OR UAV OR UAS OR DRONE OR “unmanned aerial system" OR Airplane) AND (Imaging OR Inspection OR Maintenance) AND ("Transmission Power" OR "Transmission Line" OR "Power line transmission" OR "Power Network" OR "Power line inspection" OR "Power line")

\subsection{Selection and quality criteria}

In order to support the selection of relevant studies that are aligned in the clarification of the proposed research questions, inclusion and exclusion criteria were defined. The inclusion of a study was determined by relevance, title analysis, summary, introduction, completion and when necessary by the complete reading of the study. The criteria adopted for exclusion and inclusion of the articles are presented in Tables 1 and 2. After the inclusion and exclusion criteria established, the next step was to define quality criteria. To analyze the quality of the primary studies, a list of five quality criteria for addressing the overall article has been defined and is presented in Table 3 based on those established by Kitchenham [4].

Table 1. Exclusion criteria

\begin{tabular}{ll}
\hline Code & \\
\hline EC1 & Publications that do not contain the exact key words or variations thereof will be discarded. \\
EC2 & Publications that do not use unmanned aircraft tools will be discarded. \\
EC3 & Publications that do not have the objective of monitoring transmission lines will be discarded. \\
EC4 & Publications that do not use the standalone mode will be discarded. \\
EC5 & Publications that do not have all the processes of the work or that are part of a work will be discarded. \\
EC6 & Publications that are only in planning or that have not been applied at least in the simulation environment will be discarded. \\
\hline
\end{tabular}

Table 2. Inclusion criteria

\begin{tabular}{cl}
\hline Code & Criteria \\
\hline IC1 & Publications that use unmanned aircraft for inspection on transmission lines can be selected autonomously. \\
IC2 & Publications can be selected that have different techniques for autonomous tracking of transmission lines.
\end{tabular}




\begin{tabular}{cl} 
& \multicolumn{1}{c}{ Table 3. Quality criteria } \\
\hline Code & \multicolumn{1}{c}{ Criteria } \\
\hline QC1 & Was the study described clearly and appropriately? \\
QC2 & Have the methods or techniques used in the primary studies been reported clearly? \\
QC3 & Was the virtual environment used in primary studies reported clearly? \\
QC4 & Was the study proposal evaluated/validated? \\
QC5 & Have the results been clearly stated? \\
\hline
\end{tabular}

In the criterion of note, to evaluate the articles, the scoring scale was used from 0 to 5 , being that for each criterion attended completely was added 1 point, if partially 0.5 points and if it did not meet the criterion no score was added. For cost criteria, a qualitative assessment of the materials used was established, as not all-numerical information is accessible in the publications. The scale goes from very low to very high. UAVs manufactured from scratch and/or using only one piece of equipment will be downgraded to cost. Platelet UAVs and/or equipment add-ons will be enhanced in the cost rating. It is also evaluated in this criterion the cost of maintenance and resistance of the equipment from the information of the authors themselves. In the other criteria, a general analysis is performed, measuring from the information available in the publication itself. Section 4 presents the details of the arguments made by the respective authors.

\section{CONDUCTION AND RESULTS}

The implementation of the methodology presented in Figure 2 shows that the research returned a total of 223 articles (of these, 89 IEEE articles, 65 articles by Scopus, 5 by ACM and 64 Compendex articles), as the union of these articles had duplicates (85) and articles which were not aligned with the research questions (110), it was necessary to apply a screening process to obtain only relevant articles for research.

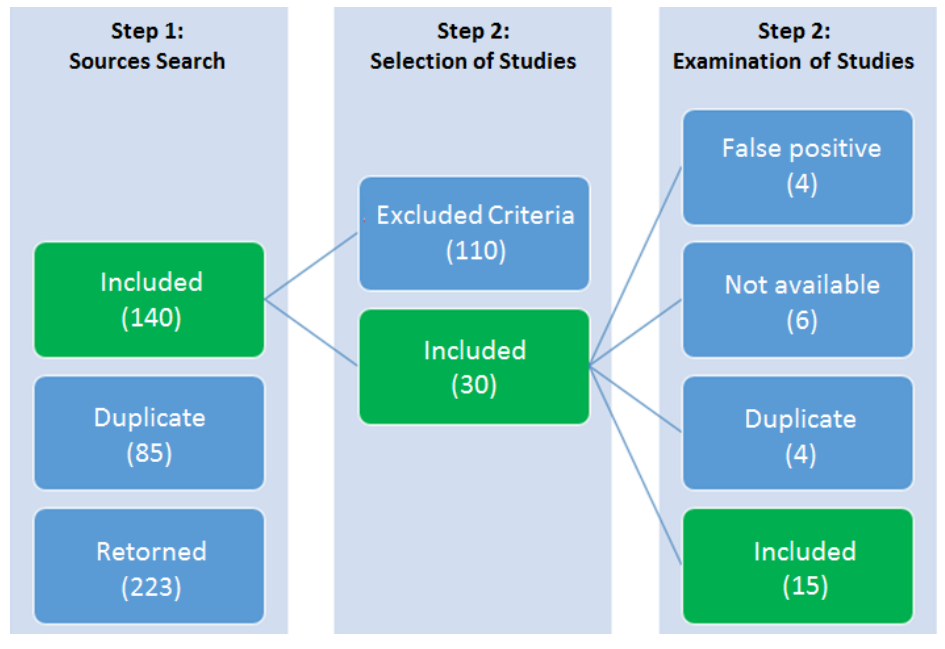

Figure 2. Selection process of primary studies

The next step was to apply the inclusion and exclusion criteria resulting in 30 articles in total and finally a more careful selection was made by reading the articles completely, removing 4 articles with duplicate content, 5 false positive articles and 6 articles that the text complete was not available. Then 15 articles were obtained which are presented in the Table 4. After the final selection of the articles, the data extraction procedure was done from the complete reading of each article. The extracted data followed the specifications described below:

- Publication data: title, author(s), publication date, publication vehicle and publication summary.

- General information: description of the objective of the article and materials used (to stipulate the cost).

- Techniques: mode used for the recognition of the transmission lines for conducting the guide.

- Results obtained: to obtain the error rates $(\%)$, the locations where the tests were run and the security issue.

- Quality criteria: note attributed from the III and additional comments from each researcher. 
Each process is performed by two or three of the authors of this paper, this allows the review of decisions and eliminates any bias that may have occurred.

The results of this SRL were presented and analyzed in order to discuss the evidence from the 15 primary studies that may answer the research questions raised. As shown in Figure 3, the IEEE and Scopus database had a high representation with nine and eight included studies respectively, as for Compendex were five primary studies the ACM had less representation with no selected study. The systematic mapping, which is the summary of the analysis performed, is presented in the Table 4.

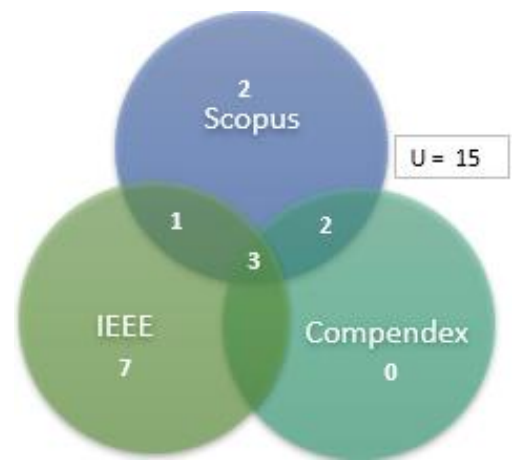

Figure 3. Quantity papers filtered on each search engine

Table 4. Characteristics identified in the articles

\begin{tabular}{|c|c|c|c|c|c|c|c|c|}
\hline Ref & Material & Methods & Tests & UAV & Country & Efficiency $(\%)$ & Cost & Quality \\
\hline [7] & Laser & Binocular vision & Real flight & Uni & China & 97 & Average & 4.0 \\
\hline$[8]$ & Camera & $\mathrm{KCF}$ tracking & Real flight & Multirotor & Uni & 90 & Low & 5.0 \\
\hline [9] & $\begin{array}{l}\text { Infrared } \\
\text { camera }\end{array}$ & GCS & Real flight & Multirotor & Uni & Uni & Average & 4.0 \\
\hline [10] & Camera & Edge detection & Real flight & Multirotor & Greece & Uni & Very low & 3.0 \\
\hline [11] & Laser & Adaptive algorithm & Laboratory & Multirotor & Uni & Uni & Average & 4.0 \\
\hline [12] & $\begin{array}{c}\text { Camera and } \\
\text { lidar }\end{array}$ & $\begin{array}{l}\text { Discrete-time } \\
\text { control }\end{array}$ & Real flight & $\begin{array}{l}\text { Hybrid } \\
\text { multirotor }\end{array}$ & Uni & Uni & Average & 4.5 \\
\hline [13] & $\begin{array}{l}\text { Magnetic } \\
\text { sensors }\end{array}$ & $\begin{array}{l}\text { Metaheuristic } \\
\text { algorithm }\end{array}$ & Laboratory & Multirotor & Uni & 95 & Average & 4.5 \\
\hline$[14]$ & Camera & Edge detection & Real flight & Multirotor & USA & Uni & Low & 4.5 \\
\hline [15] & Manifold & $\begin{array}{l}\text { Multi-sensor } \\
\text { adjustment }\end{array}$ & Real flight & Helicopter & China & 98 & $\begin{array}{l}\text { Very } \\
\text { high }\end{array}$ & 4.5 \\
\hline [16] & GPS and IMU & $\begin{array}{l}\text { Waypoints } \\
\text { adjustable }\end{array}$ & Real flight & $\begin{array}{l}\text { Fixed wing \& } \\
\text { multirotor }\end{array}$ & China & Uni & Low & 3.0 \\
\hline [17] & Camera & Edge detection & Laboratory & Uni & Uni & 97 & Average & 4.5 \\
\hline$[18]$ & Camera & Edge detection & Laboratory & Airship & Uni & Uni & Low & 3.0 \\
\hline [19] & Camera & Visual servoing & Laboratory & Multirotor & Uni & Uni & Low & 4.5 \\
\hline [20] & $\begin{array}{l}\text { Magnetic } \\
\text { sensors }\end{array}$ & $\begin{array}{l}\text { Inverse model } \\
\text { algorithm }\end{array}$ & Simulation & Uni & Uni & Uni & Low & 3.0 \\
\hline [21] & Laser & Adaptive algorithm & Laboratory & $\begin{array}{l}\text { Hybrid } \\
\text { multirotor }\end{array}$ & Uni & Uni & High & 4.5 \\
\hline
\end{tabular}

The most of articles studied proposed the use of UAV together with the camera for inspection. In general, in the article the UAV is manufactured by the team itself or with partnerships as in [9-13, 21], which provides a reduced cost and better fits the researcher's needs as presented by the authors. Was an alternative in $[8,13,14]$ adapt prefabricated or rack-mounted UAVs that generate a higher cost, mainly in maintenance. 
The authors of the articles [7, 11, 12, 15] used, in addition to the basic material, LIDAR (light detection and variation) [22], a pulsed laser optical technology that measures light reflection to obtain distance from objects, and other laser types, considerably increase the cost of the solution, but increasing the accuracy of the laser line tracking. Its direction adjustment technique is to radiate the wire horizontally, obtaining line distance information at different locations on the wire. $\mathrm{H}_{1}$ and $\mathrm{H}_{2}$ in Figure 4 are the readings of the start and end distances. The angle (1) between the sides of the section and the central axis A approximates $\alpha=0$ and $\mathrm{H}_{1}=\mathrm{H}_{2}$, so that the UAV is parallel to the wire.

$$
\alpha=\arctan \left(\left(H_{1}-H_{2}\right) /\left(H_{1}+H_{2}\right)\right) \tan \theta
$$

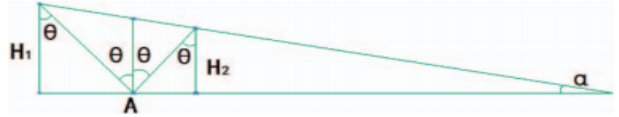

Figure 4. Schematic diagram for Yam angle measurement [7]

In [8], the Kernelized Correlation Filter (KCF) algorithm [23] was used for locating and tracking between towers, this method uses rolling matrix properties to increase processing speed. This technique is known to be efficient, but costly to track lines. In [16] a combination of various aircraft was used and tracking was performed using a global positioning system (GPS) and inertial unit of measure (IMU) waypoint, however, the article does not guarantee accurate tracking as there is influences the effect of exposure of fields near power transmission towers [24].

In [10, 14, 17-19] visual servoing type tracking [25] is used for UAV control, the method used is edge gradient detection. A smoothed image is filtered with the Sobel filter core [26] in the horizontal (Gx) and vertical (Gy) direction to obtain the first derivative (2) (3), then it traverses the pixels with a gradient vector that calculates the color intensity to identify the edge, as shown in Figure 5.

$$
\begin{gathered}
G=\sqrt{{G_{x}{ }^{2}+G_{y}{ }^{2}}^{\operatorname{Angle}(\theta)}}=\tan -1\left(G_{y} G_{x}\right) \\
=
\end{gathered}
$$

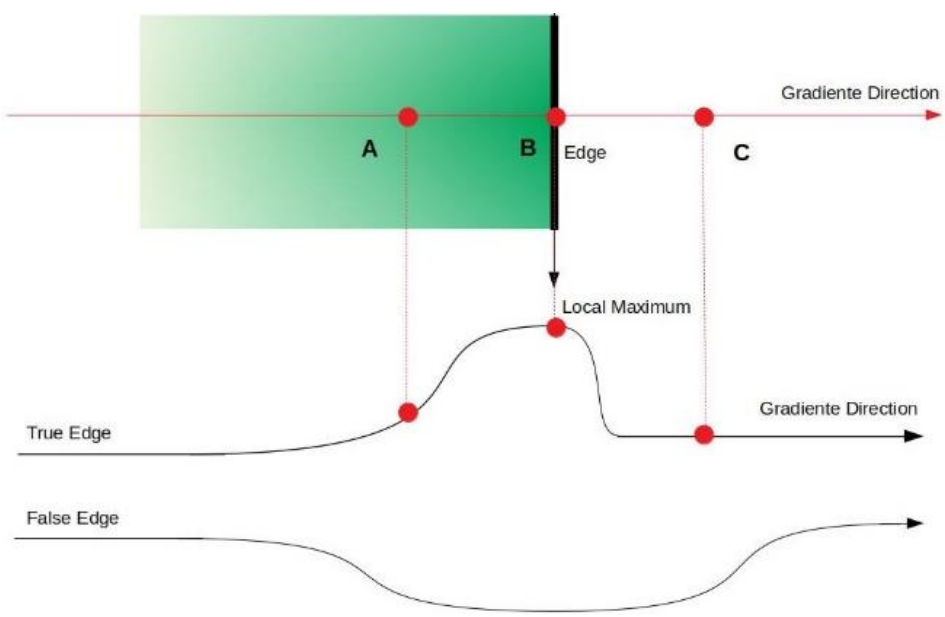

Figure 5. Canny filter gradient

Some articles use additional methods to improve the accuracy or performance of the algorithms. In [10, 17] a robust Hough transformation method [27] is used to reduce noise and identify geometric shapes in images such as lines. In $[10,14]$ also uses region of interest (ROI) separation algorithms to reduce the edge of the image on a given object, eliminating parts that should not be processed. This technique lets you explore the parameters of line continuity, similarity, and fixed spacing between power line structures.

Int J Rob \& Autom, Vol. 9, No. 4, December 2020 : 233 - 243 
It was proposed in $[13,20]$ the use of magnetic sensors, the position and current parameters of the lines were reconstructed from the magnetic field data, with an approximation model, an inverse algorithm was proposed by the author and applied in the laboratory. In the geometrical model of the straight conductor of infinite length in the plane $\mathrm{S}$ of height $\mathrm{H}$ parallel to the horizontal plane is the power transmission line. The DC current flowing in the line is indicated as I and the intensity of the magnetic field is marked as A, and a vertical line of $\mathrm{S}$ is made through $\mathrm{A}$ and crosses the plane $\mathrm{S}$ at point $\mathrm{B}$. A vertical line of $\mathrm{L}$ is made $\mathrm{B}$ to C. This model is represented in Figure 6 and the line equation in the plane (4).

$$
a x+b y+c
$$

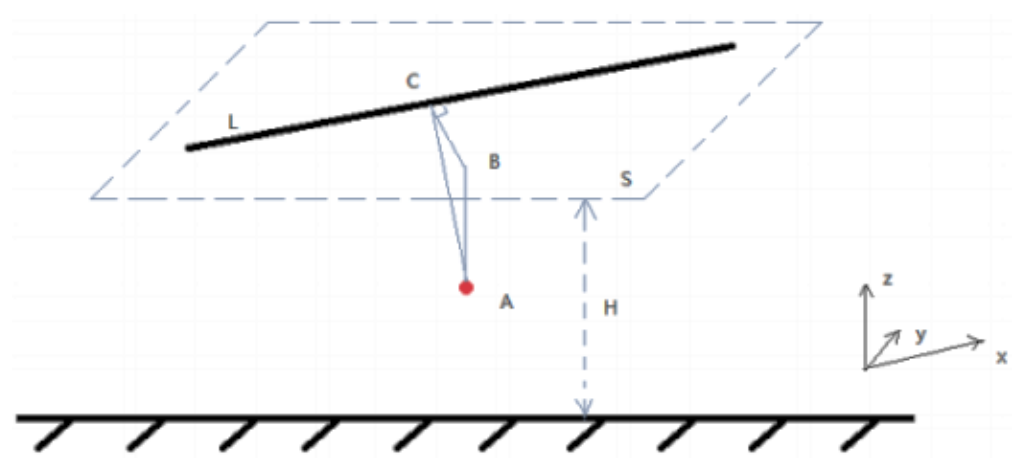

Figure 6. Transmission line model based on geometric relationship [20]

Only in $[12,16,21]$ is the energy efficiency of UAV addressed using other robot modes as shown in Figure 7. In [16] the use of different types of UAVs was discussed together and online for greater range but without losing the precision of the multirotor. In articles $[12,21]$ the authors propose the use of suspended ground wire (OGW), a line support located at the highest part of the transmission tower, thus considerably increasing the range because it does not require much effort to maintain altitude. The problem here is that many towers do not have this type of installation accessible for use.

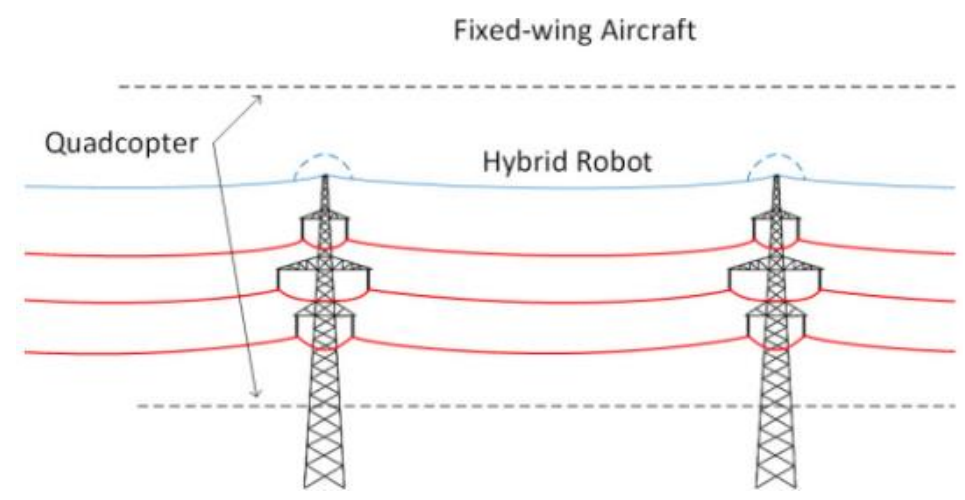

Figure 7. Workspace of different power line inspection robots [21]

\section{DISCUSSION AND IMPLEMENTATION}

In this chapter, the results and the three main research questions will be discussed. In addition, in the end a general survey of the feasibility and limitations of the studies carried out are discussed.

\subsection{Solution of questions}

SQ1: Although the articles do not present current values used in the projects, it is possible to have notion of the cost of the implementation for the solution made. Maintenance cost decreases when using self-made materials. Articles that use a few equipment, mostly a camera and a sensor are inexpensive, while those using a variety of high-end equipment have a higher cost and the results, they get are similar to those. 
SQ2: All articles the technique is considered viable and has different methods, locations and equipment not being possible direct comparison. To stipulate efficiency of the technique used was considered the information of the author himself. Overall, the error rate, when presented, is around 10\% thus demonstrating, thus considering the techniques employed as efficient. However, the energy question was little discussed, in general, the UAV had standard characteristics of autonomy.

SQ3: Due to the low error rate and the simulations or experiments carried out, it is assumed that UAVs are safe for application, but a specific approach to location and safety has not been presented in the articles. In general, the environments of use are in remote places and of little circulation of people, but in practice there are transmission lines that pass in the middle of great centers. Furthermore, no article has had a direct relationship to the safety of its implementations and equipment.

\subsection{Simulation environment}

Gazebo [28] was chosen as the simulation environment. The simulator can provide realistic feedback from the real world, with the accuracy of the dynamic environments a robot can encounter. The simulator also allows the use of angular and linear forces that can be applied to surfaces and joints to generate locomotion and interaction with the environment.

In this work, an object was used simulating a sequence of power transmission line towers of the freestanding delta model with circuit and robotic simulator UAV model Typhoon H480 with FPV, integrated with Gazebo through the PX4 simulator Figure 8. Initially, the UAV is on the ground parallel to the power transmission line, with its north to the final objective of the inspection, then to reach the desired height $\mathrm{h}$ in meters, a negative $\mathrm{z}$-axis velocity is sent for a certain time $\mathrm{t}$ in seconds, as (1).

$$
h=-z * t
$$

For line segment detection, the image needs to be properly handled. First, an image resizing to $\mathrm{W}$ (weight) 600 by $\mathrm{H}$ (height) 360 is performed, with the intention of speeding up the detection. Then the image is transformed to grayscale. The image is submitted to a Gaussian Blur filter with a Kernel value of 5, which allows highlighting the object of most interest [29].

The Canny edge detector is applied, turning the image into binary to identify the edges using neighboring pixels highlighted in white. Segmentation works with noise reduction through the Gaussian filter, then traverses the pixels with a gradient vector that calculates the color intensity to identify the edge as shown in the Figure 5.

The Hough Probabilistic algorithm is applied to this image treated with a specific region of interest, returning a list of identified segments. A filter is performed by eliminating segments with less than $25 \% \mathrm{H}$. Also, horizontal lines not in the range (6) are eliminated.

$$
160 \geq \operatorname{atan}^{2}(x, y) \cdot 180 / \pi \leq 20
$$

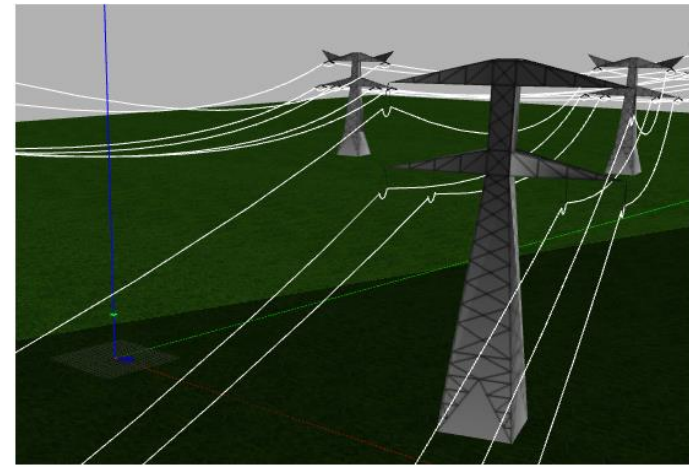

(a)

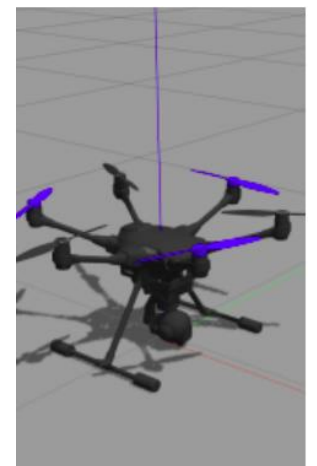

(b)

Figure 8. (a) Gazebo simulation environment and (b) UAV simulator

After obtaining the valid segments, a check is made to find the largest and closest parallel segments by comparing by simple subtraction the two end points of the line on the $\mathrm{x}$ axis only, with the same thickness, and also grouping according to position. In negative response to these characteristics, it will be considered the first segment detected. The starting and ending points of the line are then returned along with the angle 
obtained. The line segments were identified in all cases in all environments (Figure 9), thus allowing the execution of the algorithm. However, the line length was identified at $95 \%$ in the simulator [30].

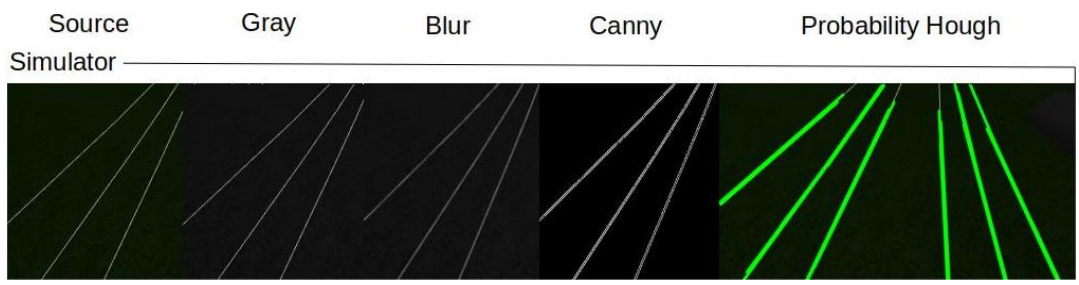

Figure 9. Image processing

\subsection{Possible lacuna analysis}

According to the information collected from the selected articles the low cost and efficient way is using a camera to use the algorithm edge detection that has little computational demand and a laser to adapt the algorithm supporting in the transmission line tracking.

The authors found that the solutions presented by the authors address the performance issue at a relatively low cost, but new embedded systems technologies for UAV appear at all times, a lacuna has been identified regarding the possibility of using deep learning with Reforcement Learning board [31]. For use on board the UAV, one can highlight the deep learning by Random Forest and Yolo, which allows for high accuracy with relatively low processing/training. These technologies can provide a generalization for different structures of towers in different environments.

For safety flight, it can be supplied with simple solutions such as parachutes, hook and protection of the propellers. It is also possible to develop redundancy systems for sensors and motors [32]. Vertical take-off and landing (VTOL) models, which are fixed wing multirotor hybrid electric UAVs, could be considered as an alternative for application by the authors for greater autonomy. Another possibility raised is the use of solar panels with [33], not necessarily for movement, but may be used for isolated components of the solution. These solutions were not presented in the articles.

\section{CONCLUSION}

Several technical means of tracking power transmission lines have been analyzed, proving to be a field that can be well explored, and with a lot of potential for development. Getting a low cost, widespread, efficient and secure solution, especially in a country with large extensions and limited financial resources in infrastructure, is a big challenge. The article in its direction obtained the resolution of the questions raised and identified possibilities not explored in the state of the art, with the focus on safety and efficiency.

It is intended by the authors the real implementation of autonomous tracking, using a university-made UAV, using solar panel, Lidar sensors and monocular camera. An adaptive algorithm for tower and line recognition will also be developed. There are reviews in the scientific literature about UAVs, however, being a systematic methodology of scientific review, no research has been found on this topic, which is very relevant in economic and social terms.

\section{ACKNOWLEDGEMENTS}

This study was financed by both Coordenação de Aperfeiçoamento de Pessoal de Nivel Superior, Brazil (CAPES) - Finance Code 001, and National Council for Scientific and Technological Development $(\mathrm{CNPq})-$ Brazil.

\section{REFERENCES}

[1] "ONS - Operador Nacional do Sistema Eltrico." http://ons.org.br/paginas/sobre-o-sin/o-sistemaem-numeros (accessed July 15, 2019).

[2] G. C. da Silva and M. Munaro, "Specifications of An Adequate System for Climbing Poles (in Portuguese: Especificações de Um Adequado Sistema Para Escalada em Postes)," Seminário Nacional de Distribuição de Energia Elétrica (SENDI), Olinda-Pernambuco, Brazil, 2008.

[3] Parsifal. https://parsif.al (accessed March 2, 2019).

[4] B. Kitchenham, "Procedures for performing systematic reviews," Keele University, Keele, UK, NICTA Technical Report 0400011T.1, 2004. 
[5] T. Dyba, T. Dingsoyr, and G. K. Hanssen, "Applying Systematic Reviews to Diverse Study Types: An Experience Report," in First International Symposium on Empirical Software Engineering and Measurement (ESEM 2007), Madrid, 2007, pp. 225-234.

[6] L. D. V. Ribeiro, et al., "Visual Servo Control in Quadrotor for Power Line Tracking," in Proceedings of 10th International Micro-Air Vehicles Conference, 2018.

[7] X. Zhang, et al., "A binocular vision based auxiliary navigation system of the unmanned aerial vehicle for power line inspection," in 2016 3rd International Conference on Systems and Informatics (ICSAI), Shanghai, 2016, pp. 291-296.

[8] X. Hui, et al., "A monocular-based navigation approach for unmanned aerial vehicle safe and autonomous transmission-line inspection," International Journal of Advanced Robotic Systems, vol. 16, no. 1, 2019.

[9] L. F. Luque-Vega, et al., "Power line inspection via an unmanned aerial system based on the quadrotor helicopter," in MELECON 2014 - 2014 17th IEEE Mediterranean Electrotechnical Conference, Beirut, 2014, pp. 393-397.

[10] A. Zormpas et al., "Power Transmission Lines Inspection using Properly Equipped Unmanned Aerial Vehicle (UAV)," in 2018 IEEE International Conference on Imaging Systems and Techniques (IST), Krakow, 2018, pp. 1-5

[11] C. Deng, J. Liu, Y. Liu and Y. Tan, "Real time autonomous transmission line following system for quadrotor helicopters," in 2016 International Conference on Smart Grid and Clean Energy Technologies (ICSGCE), Chengdu, 2016, pp. 61-64.

[12] P. Hamelin, et al., "Discrete-time control of LineDrone: An assisted tracking and landing UAV for live power line inspection and maintenance," in 2019 International Conference on Unmanned Aircraft Systems (ICUAS), Atlanta, GA, USA, 2019, pp. 292-298.

[13] Y. Wu, et al., "Overhead Transmission Line Parameter Reconstruction for UAV Inspection Based on Tunneling Magnetoresistive Sensors and Inverse Models," IEEE Transactions on Power Delivery, vol. 34, no. 3, pp. 819-827, 2019.

[14] G. Zhou, et al., "Robust real-time UAV based power line detection and tracking," in 2016 IEEE International Conference on Image Processing (ICIP), Phoenix, AZ, 2016, pp. 744-748.

[15] X. Xie, et al., "A multiple sensors platform method for power line inspection based on a large unmanned helicopter," Sensors, vol. 17, no. 6, 2017.

[16] C. Deng, et al., "Unmanned aerial vehicles for power line inspection: A cooperative way in platforms and communications," J. Commun, vol. 9, no. 9, pp. 687-692, 2014.

[17] O. A. Menendez, M. Perez, and F. A. Auat Cheein, "Vision based inspection of transmission lines using unmanned aerial vehicles," in 2016 IEEE International Conference on Multisensor Fusion and Integration for Intelligent Systems (MFI), Baden-Baden, 2016, pp. 412-417.

[18] M. Gerke and P. Seibold, "Visual inspection of power lines by U.A.S.," in 2014 International Conference and Exposition on Electrical and Power Engineering (EPE), Iasi, 2014, pp. 1077-1082.

[19] O. Araar and N. Aouf, "Visual servoing of a Quadrotor UAV for autonomous power lines inspection," in 22nd Mediterranean Conference on Control and Automation, Palermo, 2014, pp. 1418-1424.

[20] F. Gao, et al., "A novel inverse method for automatic UAV line patrolling with magnetic sensors," in 2018 IEEE International Symposium on Electromagnetic Compatibility and 2018 IEEE Asia-Pacific Symposium on Electromagnetic Compatibility (EMC/APEMC), Singapore, 2018, pp. 481-485.

[21] W. Chang, et al., "Development of a power line inspection robot with hybrid operation modes," in 2017 IEEE/RSJ International Conference on Intelligent Robots and Systems (IROS), Vancouver, BC, 2017, pp. 973-978.

[22] P. Richard, N. Pouliot, and S. Montambault, "Introduction of a LIDAR-based obstacle detection system on the LineScout power line robot," in 2014 IEEE/ASME International Conference on Advanced Intelligent Mechatronics, Besacon, 2014, pp. 1734-1740.

[23] S. Ren, et al., "Faster R-CNN: Towards real-time object detection with region proposal networks," in Advances in neural information processing systems, 2015, pp. 91-99.

[24] C. R. Paul, Introduction to electromagnetic compatibility, vol. 184, Hoboken, NJ: John Wiley \& Sons, 2006

[25] J. F. Henriques, R. Caseiro, P. Martins and J. Batista, "High-Speed Tracking with Kernelized Correlation Filters," IEEE Transactions on Pattern Analysis and Machine Intelligence, vol. 37, no. 3, pp. 583-596, 2015.

[26] I. Pitas and A. N. Venetsanopoulos, "Edge Detectors Based on Nonlinear Filters," IEEE Transactions on Pattern Analysis and Machine Intelligence, vol. PAMI-8, no. 4, pp. 538-550, July 1986.

[27] J. Canny, "A Computational Approach to Edge Detection," IEEE Transactions on Pattern Analysis and Machine Intelligence, vol. PAMI-8, no. 6, pp. 679-698, Nov. 1986.

[28] “Gazebo Simulation.” Dronecode. https://dev.px4.io/v1.9.0/en/simulation/gazebo.html (accessed May 5, 2019).

[29] W. M. Martins, et al., "A computer vision based algorithm for obstacle avoidance," Information Technology-New Generations, pp. 569-575, 2018

[30] A. J. Dantas Filho, et al., "A General Low Cost UAV Solution for Power Line Tracking," in 17th International Conference on Information Technology-New Generations (ITNG 2020), 2020, pp. 525-530.

[31] A. Carrio, et al., "A review of deep learning methods and applications for unmanned aerial vehicles," Journal of Sensors, vol. 2017, 2017.

[32] J. P. Afman, et al., "Towards a New Paradigm of UAV Safety," arXiv preprint arXiv:1803.09026, 2018.

[33] L. D. de Jesus, et al., "Greater autonomy for RPAs using solar panels and taking advantage of rising winds through the algorithm," in 16th International Conference on Information Technology-New Generations (ITNG 2019), 2019, pp. 615-616.

Int J Rob \& Autom, Vol. 9, No. 4, December 2020 : 233 - 243 


\section{BIOGRAPHIES OF AUTHORS}

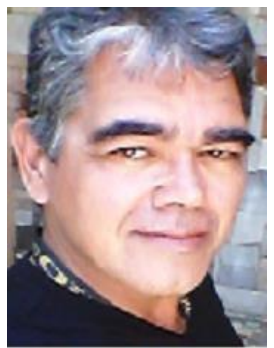

Wander M. Martins, PhD student in Electrical Engineering from the Federal University of Engineering of Itajuba, Master in Computer Science, and Technology from the Federal University of Itajuba (2018). Degree in Data Processing Technology from the Santa Rita do Sapucaí School of Administration and Informatics (1989). Data Processing Technician by the College of Application (CA) of the Itajuba Research and Education Foundation (1986). His research interests are computer vision, artificial intelligence, and remotely piloted air vehicles. His research interests are computer vision and autonomous air vehicles.

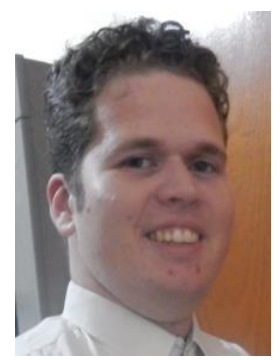

Antonio J. Dantas Filho, Bachelor of Information Systems from the Federal University of São Carlos. He holds a master student in computer science and technology from the Federal University of Itajuba. He made a computer technician at the Technical College Antonio Teixeira Fernandes. His research interests are computer vision, artificial intelligence, and remotely piloted air vehicles.

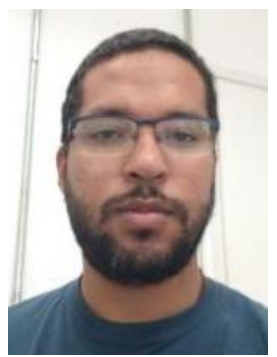

Leandro D. de Jesus graduated in Computer Science from University Paulista. He is a student of the master's degree in computer science and technology from the Federal University of Itajuba. His main research focus is UAVs.

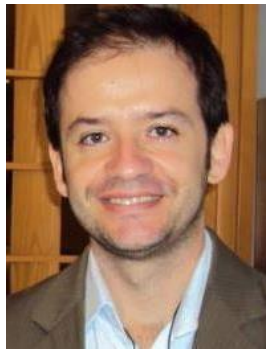

Adler D. de Souza is a PhD in Systems Engineering and Computer Science at COPPE/PESC/ UFRJ (2014), Master in Systems Engineering and Computer Science at COPPE/PESC/UFRJ (2008), Specialist in Software Process Improvement at the Federal University of Lavras - UFLA (2005) and Bachelor of Computer Science, also from UFLA (2004). He is currently engaged in research and extension projects in the areas of Software Process Improvement, Project Management, Portfolio Management, Software Factory, Value Added Analysis, Development of Serious Games for Software Engineering, Blockchain and Artificial Intelligence teaching project cost forecast and financial assets applied.

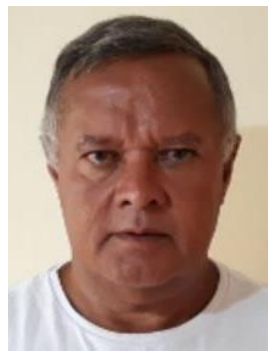

Alexandre C. B. Ramos received the B.S. in Electronic Engineering from the University of Vale do Paraiba in 1985, his M.S. and Ph.D. degrees in Electronic and Systems Engineering from Aeronautics Institute of Technology (Brazil), in 1992 and 1996, respectively. He holds Postdoctorate's studies in the Ecole Nationale de l'Aviation Civile (France) in 2013-2014. His research interests are in information systems, control of electronic processes and analytical models and simulation.

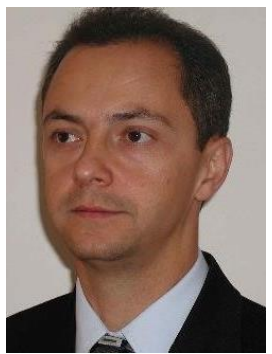

Tales C. Pimenta holds a degree in Electrical Engineering from the Federal University of Itajuba, a master's degree in Electrical Engineering from the Federal University of Itajuba and a doctorate in Electrical and Computer Engineering from Ohio University. He completed his first postdoctoral degree at The Ohio State University in 1997 on low-voltage analog integrated circuits and his second postdoctoral degree at Virginia Politechnic Institute and State University in 2005 in the field of ultra-high frequency integrated circuits, and his third postdoctoral degree $\mathrm{PhD}$ in 2014 at North Florida University in biomedical application circuits. He has experience in Electrical Engineering, focusing on Electronic Circuits, acting mainly in integrated circuits of biomedical applications. 